\title{
Structure and Composition Determination from Fluctuation X-ray Scattering
}

\author{
Dongsheng Li
}

Pacific Northwest National Laboratory, Richland, WA 99352

Since the propose of fluctuation X-ray scattering (FXS) technique developed by Kam thirty years ago[1, 2], this method has been dormant until the development of X-ray free electron laser (XFEL). In the past few years, FXS has attracted great interest $[3,4]$ when XFEL provides unprecedented beam brightness and enables recordings of single particle diffractions, opening a new field of structural determination beyond crystallography and attracts intense research activities. Advent of XFEL makes possible to expand the structure determination capability from the materials systems with periodic spatial structure to amorphous material systems, such as viruses, proteins, and biofilms. However, there are still several experimental and theoretical difficulties that hinder the development of this diffract-before-destroy technique. For example, it is technically challenging to select a single particle and align it with a tiny Xray beam and data analyses and structural reconstructions could be complicated by the lack of information on particle orientations. The taking full advantages of the short pulse and high peak brightness of XFEL promises structural determination without crystallization and maintains information content close to that from crystallography. The difficulty of targeting a single particle could be overcome in a FXS measurement by extracting structural information from scattering of a disordered ensemble of particles. Experiments on two-dimensional systems have recently been carried out on gold nano-rods and platinum coated gold nano-dumbbells demonstrating Kam's original concept of single particle structural determination by FXS.

In our seminary works, we extend Kam's theorem from homogeneous systems into heterogeneous system. We demonstrated the feasibility of structure uncovering from mixtures of identical particles. Following Kam's derivation for well-spread homogeneous particles, the resulting X-ray diffraction pattern is the superposition of those from individual particles and its signature, angular correlation, consist of two terms, the single particle auto-correlation and the interparticle cross-correlation. Averaging over a large number of angular correlation functions calculated from X-ray diffraction patterns will lead to the cancellation of the second term due to the random angular shifts and converge to the angular autocorrelation of a single particle. When adding additional species into the system while keeping the particles well dispersed, the final diffraction pattern is the sum of individual particle diffractions from all species and its angular correlation function preserves the auto- and crosscorrelations of each species with additional terms due to the intraparticle cross-correlations from different species. We find both the cross-correlations between particles of the same and distinct species cancel out by averaging a great amount of angular correlation functions and the only terms survive in the process are the individual particle auto-correlations of the same species. These auto-correlation terms are linearly proportional to their particle numbers (molar concentrations), hence the auto correlation functions of an individual species can be obtained experimentally through mixtures of various molar ratios. The structure of each kind of particles are subsequently reconstructed by applying a reverse Monte Carlo type method through iterative comparisons to minimize the target function and fit the signature angular autocorrelation

The capability in structure determination for a heterogeneous system using FXS is exemplified in a system composed of three types of nanoparticles: nanorices, nanorods and nanoprisms. The typical 
micrographs of the nanoparticle images are illustrated in the subfigures in Figure 1, which illustrate the work flow of structure determination.

In our study, FXS is also applied to determine composition of heterogeneous nanoparticle mixture when the structures of possible components are already known. Angular autocorrelation functions are taken as signatures for components. From the signature of the mixture and component signature matrix, the composition is possible to be retrieved in an inverse problem as structure determination. Furthermore, the capabilities and limitation of XFM will be discussed from the view of point resolution and noise in scattering and data analysis.

References:

[1] Kam, Z., Macromolecules, 10(5), (1977), p. 927-934.

[2] Kam, Z., M. Koch, and J. Bordas, Proceedings of the National Academy of Sciences, 78(6), (1981), p. 3559.

[3] Saldin, D., et al., Physical Review Letters, 106(11), (2011), p. 115501.

[4] Saldin, D., et al., New Journal of Physics, 12, (2010), p. 035014.

The authors acknowledge the funding support from the LDRD-funded Chemical Imaging Initiative at Pacific Northwest National Laboratory, operated for the U.S. Department of Energy by Battelle under contract DE-AC06-76RL01830.

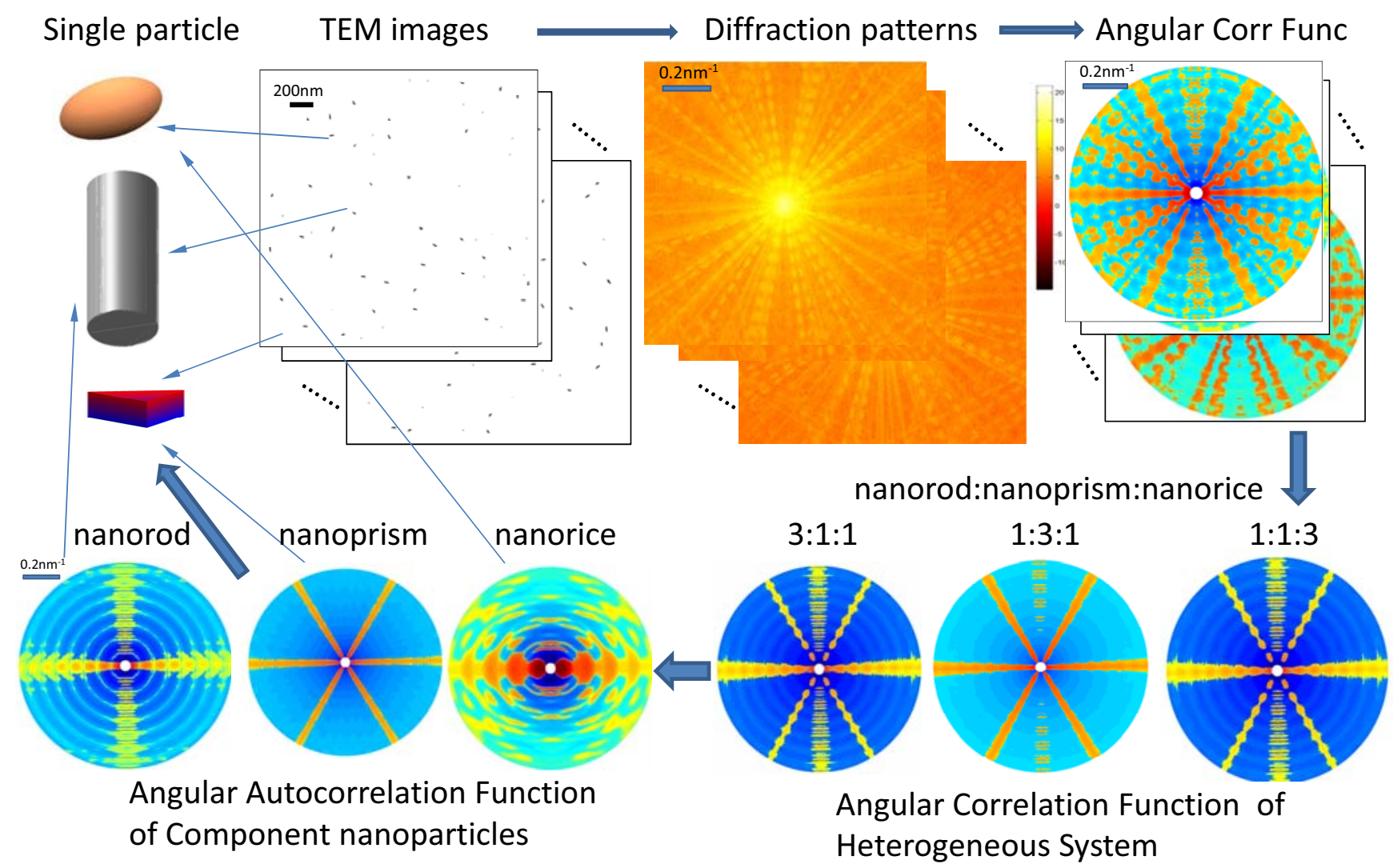

Figure 1. Scheme of single-particle structural determination from heterogeneous ensembles using fluctuation X-ray scattering technique 